Ann. Biol. anim. Bioch. Biophys., I974, 14 (4-B), 845-853.

\title{
ÉVALUATION DE DIFFÉRENTES MYCOTOXINES (AFLATOXINES, OCHRATOXINES, ZEARALENONE) SUR COUCHES MINCES PAR FLUORODENSITOMÉTRIE PAR RÉFLECTANCE
}

\author{
M. JEMMALI \\ avec la collaboration technique de M. Yvon et G. Ponce \\ Station de Biochimie et Physico-Chimie des Céréales, I. N.R.A., \\ Service des mycotoxines, \\ 16, rue Nicolas-Fortin, \\ 75013 Paris \\ RÉSUMÉ
}

La détermination de la teneur de plusieurs mycotoxines (aflatoxines, ochratoxines, zearalenone) réalisée par fluorodensitométrie par réflectance sur couche mince, est décrite et discutée. L'étude de la variabilité des mesures pour le dosage de l'aflatoxine $B_{1}$ est présentée.

\section{INTRODUCTION}

La détermination de la teneur de plusieurs mycotoxines, et en particulier de celles qui ont été mises en évidence comme polluants naturels des produits agricoles et alimentaires, s'effectue par appréciation de l'intensité de fluorescence induite par la lumière ultra-violette longue ou courte selon la nature de la toxine.

Dans la plupart des cas, la recherche et le dosage des mycotoxines consiste à extraire la toxine du produit étudié, aussi quantitativement que possible, à éliminer de l'extrait les impuretés génantes, à séparer la ou les mycotoxines par chromatographie en couche mince, enfin à les doser par évaluation de leur intensité de fluorescence après avoir confirmé leur identité.

L'estimation quantitative de la concentration de mycotoxines après chromatographie de la solution en couche mince, peut se faire soit visuellement en comparant l'intensité de fluorescence de la substance à étudier avec celle obtenue à partir d'une solution standard, soit indirectement à l'aide d'un fluorodensitomètre.

La première technique peut conduire, d'après BEckwITh et SToLoff (I968) à des erreurs d'estimation de l'ordre de $3^{\circ} \mathrm{p}$. roo dues entre autres, à l'appréciation subjective du manipulateur, à la difficulté d'évaluation de différences d'intensité 
de fluorescence et aux nombres de dilutions ou concentrations de l'extrait souvent rendues nécessaires pour ajuster la concentration en mycotoxines de l'extrait à celle du standard. Quant à la technique densitométrique, elle présente l'avantage d'être plus objective et précise, \pm 2 à 4 p. Ioo d'après Pons et al. (I 966 ) et surtout plus rapide.

Plusieurs types de fluorodensitomètres ont été décrits pour le dosage des aflatoxines, l'estimation des teneurs en toxines avec ces appareils se fait, soit par fluorodensitométrie par transmission (BECKwith et SToloff, I968), soit par fluorodensitométrie par réflectance (PONS, I97I ; BELJAARS et FABRY, I972; JEMMALI, I973).

L'objet du travail présenté dans cette note est, d'une part, d'étudier l'évaluation des différentes mycotoxines (aflatoxines, ochratoxines, zearalenone) par fluorodensitométrie par réflectance au moyen du Vitatron TLD roo, et, d'autre part, d'estimer les variations de teneurs en toxines entre plaques et les variations de teneurs en toxine sur une même plaque. Ces estimations sont effectuées pour l'aflatoxine $B_{1}$ seulement.

\section{MATÉRIEL ETT MÉTHODES}

\section{I. - Matériel d'étude utilisé}

Appareillage et réactifs utilisés.

- Fluorodensitomètre (1).

Le schéma de l'appareil est représenté à la figure I.

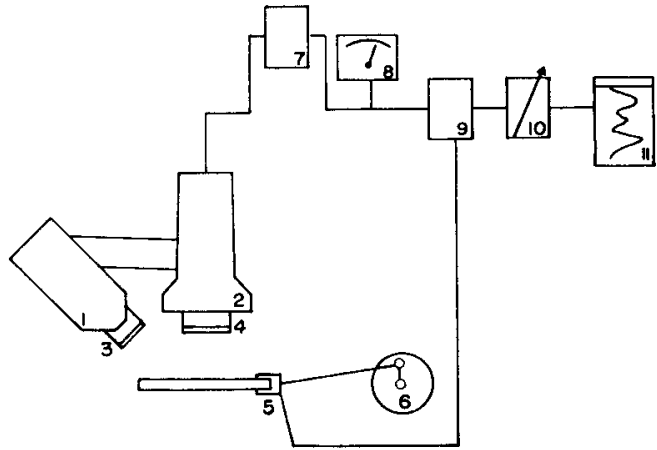

FIG. I. - Schéma du Vitatron TLD 100

I : Source de lumière avec lampe $\mathrm{Hg}$

2 : Photomultiplicateur

3 : Filtre primaire

4: Filtre secondaire

5 : Table d'échantillons

6 : Excentrique
7 : Amplificateur linéaire

8: Mètre enregistreur

9 : Intégrateur

Io : Amplificateur variable

II : Enregistreur avec intégrateur

\section{- Mycotoxines standards:}

Aflatoxines $\mathrm{B}_{1}, \mathrm{~B}_{2}, \mathrm{G}_{1}, \mathrm{G}_{2}\left({ }^{2}\right)$;

Ochratoxines A, B ( ${ }^{(3)}$;

Zearalenone $\left({ }^{4}\right)$.

(1) Vitatron TLD Ioo.

(2) Produits Makor chemicals,

(3) Fournis aimablement par Dr CAMPBELI (F. D. A.).

(4) Fournis aimablement par Dr MrRochA (St Paul).

(b) Spectronic 505 Bausch et Lomb. 
Le tableau I donne les caractéristiques physico-chimiques ayant servi à déterminer les concentrations des solutions de mycotoxines par spectrophotométrie $\left(^{5}\right)$.

\section{TABLEAU I}

Caractéristiques des mycotoxines étudiées

\begin{tabular}{|c|c|c|c|c|c|c|c|}
\hline \multirow[t]{2}{*}{ Mycotoxines } & \multicolumn{4}{|c|}{$\begin{array}{c}\text { Aflatoxines }(1) \\
\text { dans benzène-acétonitrile }(98 / 2 \mathrm{v} / \mathrm{v})\end{array}$} & \multicolumn{2}{|c|}{$\begin{array}{l}\text { Ochratoxines }\left({ }^{2}\right) \\
\text { dans benzène- } \\
\text { acide acétique } \\
(99 / 1 \mathrm{v} / \mathrm{v})\end{array}$} & \multirow[t]{2}{*}{$\begin{array}{c}\text { Zearalenone }\left({ }^{3}\right) \\
\text { dans } \\
\text { alcool absolu }\end{array}$} \\
\hline & $\mathrm{B}_{1}$ & $\mathrm{~B}_{2}$ & $\mathrm{G}_{1}$ & $\mathrm{G}_{2}$ & A & $\mathrm{B}$ & \\
\hline $\begin{array}{l}\text { Poids moléculaire ........ } \\
\text { en nm (maximum d'ab- } \\
\text { sorption) } \ldots \ldots \ldots \ldots \ldots \\
\text { Coefficient d'extinction.... }\end{array}$ & $\begin{array}{r}312 \\
\\
348 \\
19800\end{array}$ & $\begin{array}{r}314 \\
348 \\
20900\end{array}$ & $\begin{array}{r}328 \\
\\
355 \\
17100\end{array}$ & $\begin{array}{r}355 \\
18200\end{array}$ & $\begin{array}{r}333 \\
5550\end{array}$ & $\begin{array}{r}369 \\
\\
320 \\
6000\end{array}$ & $\begin{array}{r}318 \\
274 \\
13909\end{array}$ \\
\hline $\begin{array}{r}\text { Concentrations en } \mu g / m l \\
\text { des solutions utilisées }\left({ }^{4}\right)\end{array}$ & $\begin{array}{l}0,97 \\
0,72\end{array}$ & 0,57 & 0,65 & 0,62 & 11,9 & 23,9 & 13,2 \\
\hline
\end{tabular}

(1) (Rodricks et Stoloff, 1970).

(') (SToloff et al., 1971).

(') (URRY et al., 1966).

(4) Les solutions de mycotoxines correspondent à l'optimum de concentration pour l'obtention de bonnes mesures.

- matériel courant de chromatographie couche mince ;

- plaques chromatographie couche mince de gel de silice MN-GHR ;

- solvants de développement des différentes mycotoxines résumés sur le tableau 2.

\section{TABLEAU 2}

Solvants de développement utilisés pour les différentes mycotoxines

\begin{tabular}{c|c}
\hline Solvants & Mycotoxines \\
\hline $\begin{array}{c}\text { Éther-méthanol-eau } \\
(96 / 3 / 1 \mathrm{v} / \mathrm{v})\end{array}$ & $\begin{array}{c}\text { Aflatoxines } \\
\mathrm{B}_{1} \mathrm{~B}_{2} \mathrm{G}_{1} \mathrm{G}_{2}\end{array}$ \\
\hline $\begin{array}{c}\text { Toluène-acétate d'éthyle-acide } \\
\text { formique }(6 / 3 / 1 \mathrm{v} / \mathrm{v})\end{array}$ & $\begin{array}{c}\text { Ochratoxines } \mathrm{A}, \mathrm{B} \\
\text { Zearalenone }\end{array}$ \\
\hline \hline
\end{tabular}

\section{II. - Méthode utilisée}

Mode opératoire.

Des quantités connues et croissantes de mycotoxines sont déposées sur plaque à l'aide d'une seringue Hamilton. Après développement du chromatogramme on procède à la lecture des taches au fluorodensitomètre en utilisant des filtres convenables pour chaque groupe de toxines (tabl. 3).

Pour l'étude des variations interplaques et intraplaque, effectuée seulement avec l'aflatoxine 
TABLEAU 3

Filtres en nm utilisés pour les mycotoxines étudiées

\begin{tabular}{l|c|c|c}
\hline \hline & Filtres & Mycotoxines & \\
\hline & Aflatoxines & Ochratoxines & Zearalenone \\
Primaire & 365 & 332 & $313^{*}$ \\
Secondaire & 443 & 475 & 443 \\
\hline
\end{tabular}

* En raison des difficultés d'obtenir une raie à 313 nm, différentes combinaisons des filtres ont été testées ; c'est l'association d'un filtre liquide L 313 à un filtre $\mathrm{B}(\mathbf{1})$ qui nous a donné satisfaction.

(1) Vitatron.

$\mathbf{B}_{1}$, on dép ose sur cinq plaques couche mince de silica gel des concentrations croissantes en toxine, de plus on a déposé sur une même plaque plusieurs fois deux différentes concentrations d'aflatoxine $B_{1}$.

\section{Expression des résultats.}

Pour toutes les mycotoxines étudiées, un diagramme d'étalonnage est établi en portant les valeurs des nombres d'impulsions représentant les surfaces des pics correspondant à l'intensité de chaque tache en fonction des teneurs en mycotoxines. Pour l'aflatoxine $B_{1}$ une analyse de variance est effectuée sur les résultats entre plaques et à l'intérieur d'une plaque.

\section{RÉSULTATS}

\section{A. - Courbes d'étalonnage des différentes toxines}

Afin d'éprouver le degré de liaison entre les 2 facteurs (nombre d'impulsions, $y$, et concentrations en toxines, $x$ ) nous avons calculé le coefficient $r$ pour chacune des courbes d'étalonnage. Les résultats (tabl. 4) montrent une liaison hautement significative entre les teneurs en toxines et le nombre d'impulsions.

\section{TABLEAU 4}

Coefficients $r$ relatifs au degré de liaison entre les concentrations en toxines et le nombre d'impulsions pour les différentes mycotoxines

\begin{tabular}{c}
\hline Mycotoxines \\
Aflatoxines
\end{tabular}$\left\{\begin{array}{l}\mathrm{B}_{1} \\
\mathrm{~B}_{2} \\
\mathrm{G}_{1} \\
\mathrm{G}_{2}\end{array}\right.$


De plus, la figure 2 montre que cette liaison est linéaire pour toutes les toxines dans le domaine considéré.

Par ailleurs, on constate que pour une même teneur en toxines, les intensités de fluorescence des aflatoxines se classent par ordre décroissant d'intensité comme suit : $B_{2}>B_{1}>G_{2}>G_{1}$. Ces valeurs ne sont pas en accord avec celles relevées dans la littérature (Pons et al., I966) donnant des intensités de fluorescence dans l'ordre suivant: $B_{2}, G_{2}, B_{1}, G_{1}$. Ces résultats différents s'expliquent par le fait que les intensités de fluorescence sont, en particulier, fonction de la nature du silica gel employé pour les plaques (pureté, granulométrie), des filtres secondaires utilisés et des particularités du fluorodensitomètre employé (RodRICKs, I973; JemmaLI, I972).

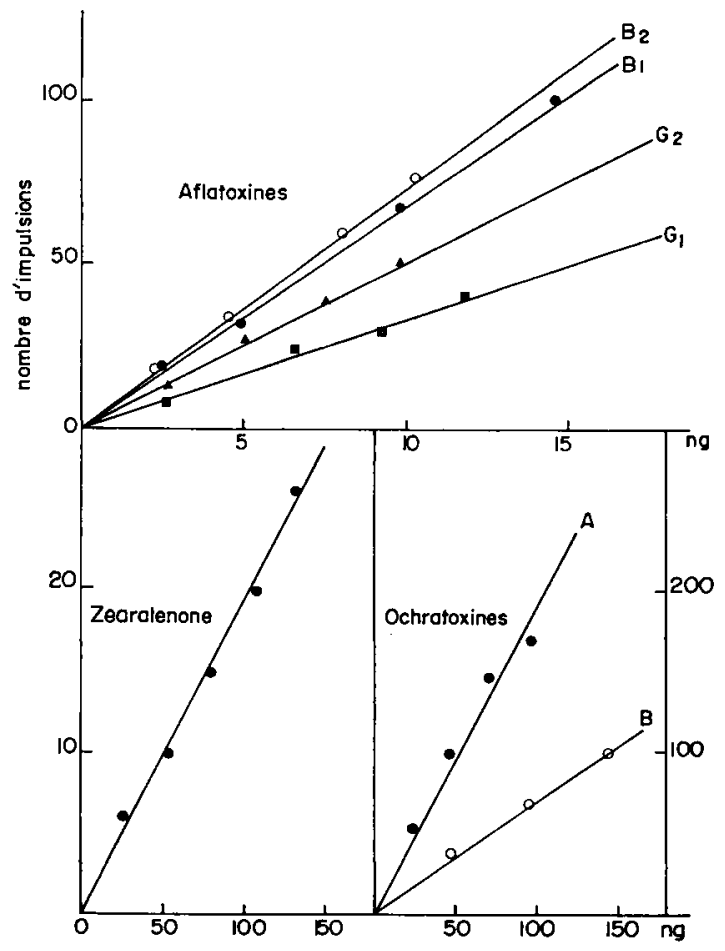

FIG. 2. - Courbes d'étalonnage des différentes mycotoxines étudiées

Notons que les intensités de fluorescence des 4 aflatoxines déterminées en milieu liquide sont également différentes selon que le solvant utilisé est du méthanol, de l'éthanol ou du chloroforme (Pons et al., r969).

L'intensité defluorescence dess différentes aflatoxines doit être précisée pour chaque lot de silice utilisé. Dans le cas des ochratoxines, la fluorescence de A est supérieure à celle de $B$.

\section{B. - Dosage des aflatoxines : précision de mesures}

Les résultats relatifs à l'étude des variations interplaques et intraplaque sont rassemblés dans les tableaux 5 et 6 . On constate, après analyse de variances :

- que les variations interplaques mettant en jeu les erreurs dues à l'effet plaque, 


\section{TABLEAU 5}

Évaluation de l'aflatoxine $B_{1}(0,97 \mu \mathrm{g} / \mathrm{ml})$ à l'aide d'un fuorodensitomètre par réflectance. Étude de la variation interplaques (reproductibilité)

Plaques MN. G-HR

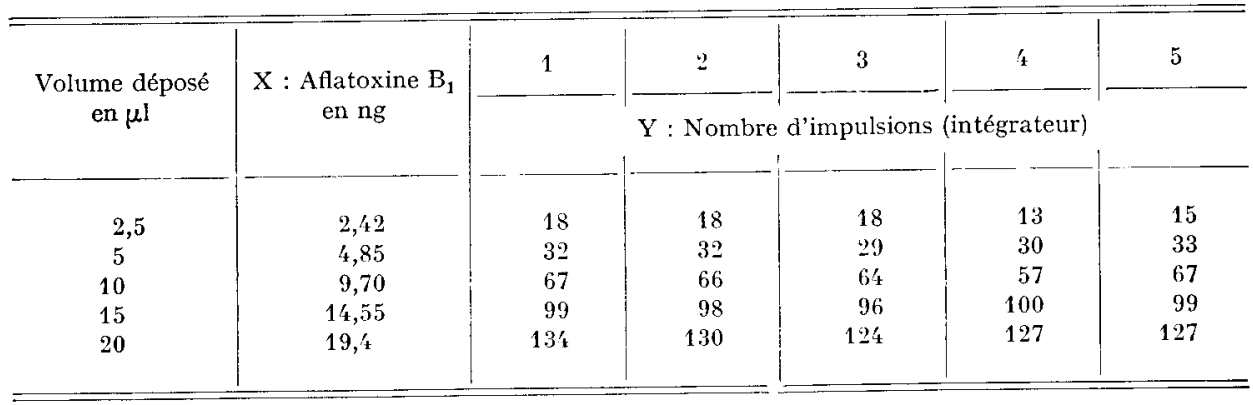

Analyse de variance

\begin{tabular}{l|r|r|r|c}
\hline Source de variations & Somme des carrés & DL & Carré moyen & F \\
\hline & $71,4^{\prime}$ & & 17,86 \\
Plaques & 43015,84 \\
Concentrations & 97,76 & 4 & 6,11 & 2,92 \\
Erreur & 16 & 4
\end{tabular}

A 5 p. $100 \mathrm{~F}_{16}^{4}=3,01 \quad \mathrm{~F}=2,92 \quad \mathrm{~F}_{16}^{4}:$ Facteur plaque non significatif.

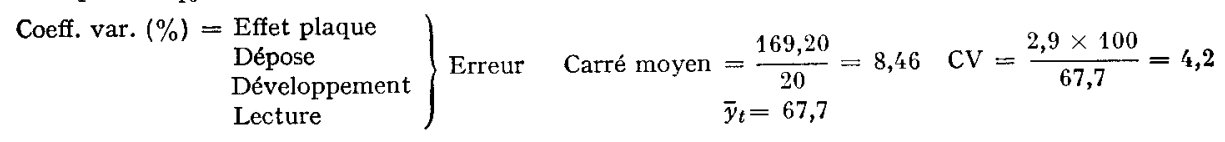

\section{TABLEAU 6}

Évaluation de l'aflatoxine $B_{1}\left(0,7^{2} \mu \mathrm{g} / \mathrm{ml}\right)$ à l'aide d'un fuorodensitomètre par réflectance. Étude de la variation intraplaque (répétabilité)

Plaque MN. G-HR

\begin{tabular}{|c|c|c|c|c|c|c|c|c|c|c|c|}
\hline Volume déposé & \multicolumn{6}{|c|}{$2,5 \mu l$} & \multicolumn{5}{|c|}{$20 \mu 1$} \\
\hline$x:$ Aflatoxine $\mathrm{B}_{1}$ en $\mathrm{ng}$ & 1,8 & 1,8 & 1,8 & & 1,8 & 1,8 & 14,4 & 14,4 & 14,4 & $14,4_{1}$ & 14,4 \\
\hline \multirow[t]{3}{*}{$\begin{array}{c}y: \text { Nombre d'impulsions } \\
\quad \text { (intégrateur) }\end{array}$} & 12 & 12 & 13 & & 12 & 12 & 99 & 98 & 99 & 100 & 100 \\
\hline & & ff. v & $\begin{array}{r}\mathrm{S}^{2} \\
\mathrm{~S} \\
\vec{y} \\
(\%)\end{array}$ & & $\begin{array}{r}0,2 \\
0,4 \\
12,2 \\
3,6\end{array}$ & & & Coeff. v & $\begin{array}{r}\mathrm{S}^{2} \\
\mathrm{~S} \\
\bar{y} \\
\text { Ir. }(\%)\end{array}$ & $\begin{array}{l}= \\
= \\
=9 \\
=\end{array}$ & \\
\hline & & & & & & var. & $\begin{array}{l}S_{t}^{2}= \\
S_{t}= \\
\bar{y}_{t}= \\
\%_{0}=\end{array}$ & $\begin{array}{c}0,45 \\
0,67 \\
55,7 \\
1,2\end{array}$ & & & \\
\hline
\end{tabular}


à la dépose, au développement des plaques et à la lecture, entraînent une erreur totale de l'ordre de 5 p. Ioo exprimée en terme de coefficient de variation;

- que les variations intraplaque sont peu importantes, elles sont de l'ordre de I,5 p. Ioo dans l'intervalle considéré.

A partir de cette étude, il était également intéressant de faire une évaluation statistique de la précision sur la base d'un étalonnage. Il suffisait alors d'effectuer une mesure par valeur de concentration et d'exploiter statistiquement ces résultats en utilisant la méthode des moindres carrés.

A partir des valeurs obtenues sur une plaque (plaque $n^{\circ} \mathrm{I}$ ) nous avons calculé la droite de régression extimée de $y$ en $x$

$$
y=a x+b=6,863 x+0, \operatorname{IO}_{2}
$$

La formule de propagation des erreurs permet de déduire pour une valeur $x_{0}$ donnée l'intervalle de confiance pour $y_{0}$

$$
y_{0} \pm t_{s} \sqrt{\mathbf{I}+\frac{\mathbf{I}}{n}+\frac{\left(x_{0}-\bar{x}\right)^{2}}{\mathrm{~S} x^{2}}}
$$

l'intervention du terme en $\frac{I}{n}$ provient de l'erreur sur l'estimation du point moyen $(\bar{x}, \bar{y})\left(n:\right.$ nombre de couples de points pour tracer la droite), celle du terme $\left(\frac{\left(x_{0}-\bar{x}\right)^{2}}{\mathrm{~S} x^{2}}\right)$ provient de l'erreur sur l'estimation de la pente de régression. L'erreur qui en résulte sur la prévision est d'autant plus grande que le point $x_{0}$ concentration étudiée_est

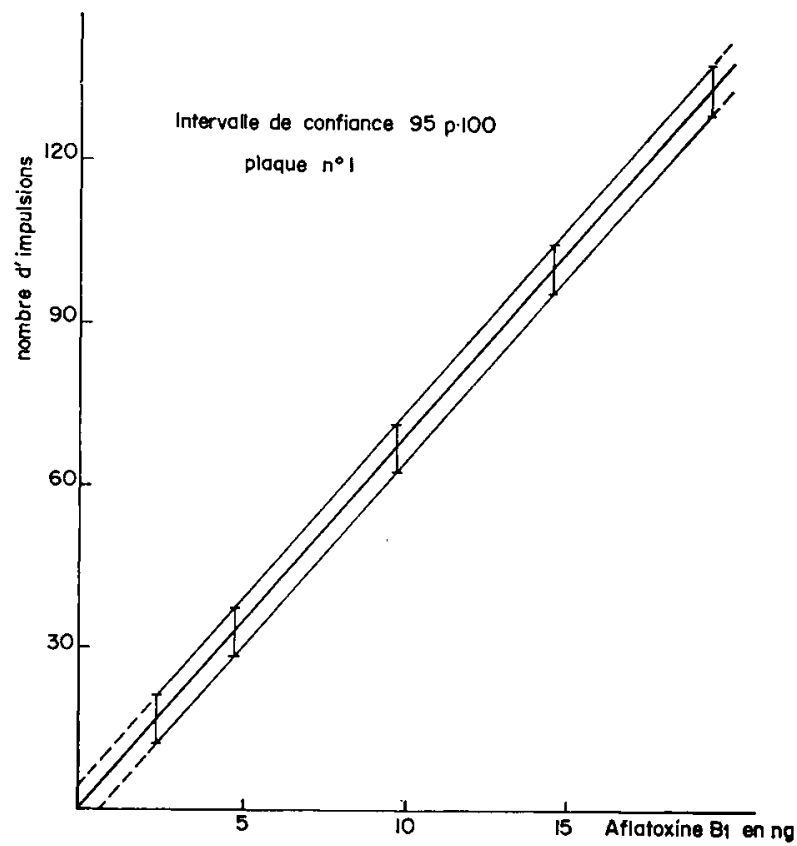

FıG. 3. - Droite de régression pour l'aflatoxine $B_{1}$ et courbes représentatives des intervalles de confiance 
plus loin du point moyen. Quand $x_{0}$ varie, les limites de confiance de $y_{0}$ correspondant décrivent les deux courbes représentatives (fig. 3) de l'équation ( $\mathrm{I}$ ), $t=2,8$ pour 3 degrés de liberté avec un risque de 5 p. Ioo.

Pour un $y_{0}$ donné, la valeur de $x_{0}$ correspondante se lit sur la même droite de régression et son intervalle de confiance se détermine à partir des courbes tracées. La valeur numérique de l'intervalle de confiance est :

$$
x_{0} \pm \frac{t_{s}}{a} \sqrt{\mathbf{I}+\frac{\mathbf{I}}{n}+\frac{\left(y_{0}-\bar{y}\right)^{2}}{a^{2} \mathrm{~S} x^{2}}}
$$

\section{CONCLUSION}

La fluorodensitométrie par réflectance constitue une méthode de choix, dans le domaine d'application étudié, pour l'estimation quantitative des mycotoxines. Compte-tenu de l'appareil utilisé, la meilleure sensibilité se situe entre 2 et I5 ng pour les aflatoxines, Io et roo ng pour l'ochratoxine $\mathrm{A}$, I5 et I5o ng pour l'ochratoxine B, 25 et $150 \mathrm{ng}$ pour la zearalenone.

$$
\text { Reçu pour publication en mai } 1974 .
$$

\section{REMERCIEMENTS}

Cette étude est réalisée grâce au concours financier de l'I. N. S. E. R. M. Nous remercions MM. Melcion et Drevet (I. N. R. A.) de nous avoir permis d'utiliser le matériel de calcul statistique.

\section{SUMMARY}

\section{EVALUATION OF DIFFEREN'T MYCOTOXINS (AFLATOXINS, OCHRATOXINS, ZEARAIENONE) ON THIN LAYER PLATES BY REFLECTANCE FLUORODENSITOMETRY}

A technic of quantitative evaluation of different mycotoxins (aflatoxins, ochratoxins, zearalenone) on thin layer chromatography is described and discussed. A critical study of determination of aflatoxin $B_{1}$ is presented and discussed according to variance analysis.

\section{RÉFÉRENCES BIBLIOGRAPHIQUES}

Beckwith A. C., Stoloff L., 1968. Fluorodensitométrie measurements of aflatoxin thin layer chromatograms. J. Assoc. Offic. Anal. Chemists, 51, 602-608.

Beljaars P. R., Fabry F. H., Pickott M. M. A., Peeters M. J., 1972. Quantitative fluorodensitometric measurement of aflatoxin $\mathrm{B}_{1}$ with a flying spot Densitometer. J. Ass. Offic. Anal. Chemists, 55, I3IO-I3I5.

Jemmali M., 1973. Collaborative studies on the determination of aflatoxins in peanuts products in France. Pure Applied Chem., 35, 267-270.

Jemmali M., 1972. Résultats non publiés.

Pons W. A. Jr, Robertson J. A., Goldblatr L. A., I966. Objective fluorometric measurement of aflatoxins on TLC plates. J. Am. oil chemist's soc., 43, 665-669. 
Pons W. A. Jr., Goldblatt L. A., I969. Physicochemical assay of aflatoxins. Aflatoxin Edited by Goldblatt L. A. Academic Press.

PoNs W. A. Jr., I97I. Évaluation of reflectance fluorodensitometry for measuring aflatoxin on thin layer plates. J. Assoc. Offic. Anal. Chemists, 54, 870-873.

Ropricks J. V., StolofF L., I97o. Determination of concentration and purity of aflatoxin standards. J. Ass. Offic. Anal. Chemists, 53, 92-95.

RoDricks J. V., I973. Communication personnelle.

Stoloff L., Nesheim S., Yin L., Rodricks J. V., Stack M., Campbell A. D., r97i. A multimycotoxin detection method for aflatoxins, ochratoxins, zearalenone, Sterigmatocystin and Patulin. J. Assoc. Off. Anal. Chemists, 54, 9I-97.

UrRy W. H., Wehrmeister H. L., Hodge E. B., Hidy P. H., ig66. The structure of zearanelone. Tetrahedron Latt, 27, 3I09-3I I 4 . 